andererseits $\operatorname{der} \beta$ - und $\gamma$-Bereich als verhältnismäßig quantitativ gering aufzeigen.

Nähere Aussagen ohne Absorption bzw. Identitätsreaktionen mit isolierten Komponenten wären hier spekulativ. Es müßten sich biochemische bzw. organ- analytische Untersuchungen anschließen um die genauere Aufklärung eines Milzextraktes zu ermöglichen und um die Frage zu klären, inwieweit spezifische Identitätsreaktionen zwischen Serumeiweißkörpern und Organeiweißkörpern vorliegen.

\title{
Literatur
}

1. Götz, H. und F. Scheiffarth, Klin. Wschr. 41, 587 (1963). 2. Götz, H., F. Scheiffarth und I. Dübeler, Gastroenterologia, Basel 98, 30 (1962). - 3. Scheiffarth, F., H. Götz und G. Schernthaner, Clin. chim. Acta (Amsterdam) 6, 481 (1961). 4. Scheiffarth, F., H. Götz und G. Thuma, Clin. chim. Acta (Amsterdam) 7, 355 (1962). - 5. Götz, H., G. Berg und F. SCheIfFARTH, Zschr. Immunit.forsch., Jena 114, 72 (1962). 6. Grabar, P., Bull. Soc. Chim. biol. 36, 65 (1954); Dtsch. med. Wschr. 79, 1916 (1954); Zbl. Bakteriol. 164, 15 (1955). - 7. Grabar, P. und P. Burtin, Analyse immuno-électrophorétique. Application avec liquides biologiques humains. Masson \& Cie., Paris (1960). - 8. Grabar, P. und C. A. Williams, Biochim. biophysica Acta (Amsterdam) 10, 193 (1953); 17, 67 (1955). 9. Scheidegger, J. J., Internat. Arch. Allergy 7, 103 (1955). 10. Williams, C. A. und P. Grabar, J. Immunol., Baltimote 74,
158 (1955). - 11. Uriel, J. und P. Grabar, Ann. Inst. Pasteur, Paris 90, 429 (1956). - 12. URIEL, J. und J. J. Scheideggrr, Bull. Soc. Chim. biol. 37, 165 (1955). - 13. Scheifrarth, F., H. GötZ und H. KNopfF, Acta haemat. 26, 169 (1961). - 14. Uriel, J., P. Grabar und Ch. Wunderly, Clin. chim. Acta (Amsterdam) 2, 35 (1957). - 15. Scheiffarth, F., H. Götz und F. CzAGANY, Med. Welt 28, 1449 (1961). - 16. URIEL, J., Bull. Soc. Chim. biol. 39, Suppl. I, 105 (1957); Clin. chim. Acta (Amsterdam) 3, 17 (1958); 3, 384 (1958). - 17. ScheIfFARTH, F., H. Warnatz und H. Götz, Med. Welt 43, 2216 (1961). - 18. HeInkel, K., in: N. Henning, Klinische Laboratoriumsdiagnostik, 2. Aufl., S. $431 \mathrm{ff}$., Urban \& Schwarzenberg, München-Berlin (1960). - 19. ScheIfFARTh, F. und H. Götz, Klin. Wschr. 42, 130 (1964).
Professor Dr. F. Scheiffarth Medizinische Universitätsklinik 852 Erlangen, Krankenhausstr. 12

\section{Zur Bestimmung des Pregnandiols im Harn nach KLOPPER, MICHIE und BROwN ${ }^{1}$ )}

Von

\author{
G. E. HALDER
}

\author{
Aus dem Laboratorium der Frauenklinik des Städt. Krankenbauses im Friedricbshain \\ (Seiner Zeit Dirig. Arat: Prof. Dr. Dr. W. Pschyrembel)
}

(Der Schriftleitung zugegangen am 12. November 1963)

\begin{abstract}
In Anbetracht der weiten Verbreitung, die die KLoppersche Methode zur quantitativen Bestimmung des Pregnandiols im Harn gefunden hat, wird über eigene Erfahrungen bei der routinemäßigen Durchführung dieser Bestimmungen berichtet. Neben einigen Modifikationen wird insbesondere die Anwendung der AlLENschen Korrektur bei der Messung der Pregnandiol-Farblösungen empfohlen. An 66 Harnen wurde sowohl die monochromatische Messung bei $430 \mathrm{~m} \mu$ wie die Allensche Korrektur bei 380, 420 und $460 \mathrm{~m} \mu$ durchgeführt. Hierbei werden gegenüber der Originalmethode im Mittel um 28\% niedrigere Pregnandiol-Ausscheidungen gemessen. Eine Abkürzung der 17-stdg. Entwicklung des Pregnandiol-Farbkomplexes ist nicht möglich.

In view of the widespread use of KLOPPER's quantitativ determination of pregnandiol in urine, results from the routine use of the method in this laboratory are reported. Some modifications and especially the use of ALLEN's correction in measuring the pregnandiol colour are recommended. Both monochromatic measurements at $430 \mathrm{~m} \mu$ and AlLEN's correction at 380,420 and $460 \mathrm{~m} \mu$ were performed on 66 urines. The measured pregnandiol excretions were on an average $28 \%$ lower than shown by the original method. The 17 hours development time of the pregnandiolcolour complex could not be shortened.
\end{abstract}

Die von Klopper, Michie und Brown (1) im Jahre 1955 publizierte Methode zur quantitativen Bestimmung des Pregnandiols im Urin erwies sich als das

1) Diese Arbeit wurde in den Jahren 1960/61 durchgeführt. z. Z. genaueste und empfindlichste Verfahren. Sie löste ältere Methoden, insbesondere die von VenNing angegebene ab $(2,3)$. Mit dieseñ Verfahren war eine Erfassung geringer Pregnandiolgehalte nur mögliç, gesammelt und aufgearbeitet wurden $(4,5)$. Die von 
wenn große Harnmengen, d. h. mehrere Tagesharne Westphal (6) eingeführte Fällung des Pregnandiols als Bariumsalz erhöhte zwar die Empfindlichkeit des Nachweises sehr, konnte die Spezifität jedoch nicht wesentlich steigern. Die Erfassung z. B. der in der Proliferationsphase des Zyklus ausgeschiedenen Pregnandiolmengen war mit diesen Fällungsmethoden nicht möglich. Auch die von Astwoon und Jones (7) publizierte Modifikation, Fällung des durch Hydrolyse aus dem Glucuronidkomplex freigesetzten Pregnandiols mit Äthanol, änderte diesen Tatbestand nicht. Erst die von TALBOT (8) eingeführte kolorimetrische Messung der Gelbfärbung des Pregnandiols, die bei Umsetżung mit konz. Schwefelsäure auftritt, legte die Grundlage zu empfindlicheren Nachweismethoden. Sommerville, Gougr und MARRIAN (9) konnten Genauigkeit und Reproduzierbarkeit so weit erhöhen, daß trotz der Unzulänglichkeiten, auf die besonders SMITH hinwies (10), dieses kolorimetrische Verfahren bis zum Jahre 1955 das zuverlässigste und genaueste darstellte. Die weite Verbreitung, die die dann im Jahre 1955 von KLOPPER angegebene Methode inzwischen gefunden hat, veranlaßt uns, hier über unsere Erfahrungen und einige Modifikationen zu berichten. Dabei wird die Originalmethode, an der vor allem die zweimalige chromatographische Reinigung der zur Kolorimetrie kommenden Pregnandiolextrakte neu ist, als bekannt vorausgesetzt.

\section{Versuche}

Hydroly'se: Wir setzen grundsätzlich $1 / 10$ der 24-Stdn.-Harnmenge ein, da dann in jedem Fall kräftige Farblösungen erhalten werden. Nur in Ausnahmefällen liegen die Extinktionen so hoch, daß mit Küvetten geringerer Schichtdicke gearbeitet werden muß (vgl. unten).

Extraktion: Wir extrahieren stets mit vorgewärmter und kochsalzgesättigter Natronlauge. So treten kaum noch störende Emulsionen auf.

Oxydation: Auch die Kaliumpermanganatlösung zur Oxydation wird, in 1 Natronlauge frisch angesetzt, auf dem Wasserbade vorgewärmt. Dadurch exzielt man eine rasche und vollständige Oxydation. Man schüttelt bis zur vollständigen Entfärbung der $\mathrm{KMnO}_{4}$-Lösung und muß auf vollständiges Abfiltrieren des ausfallenden Braunstein-Niederschlages achten. Die $\mathrm{KMnO}_{4}$-Qualität ist von untergeordneter Bedeutung, wie ein Versuch, bei dem wir $\mathrm{KMnO}_{4}$ (Merck) und $\mathrm{KMnO}_{4}$ (Handelsware) eingesetzt haben, zeigte. Die maximale Differenz, die wir hierbei beobachteten, betrug 3,1\% im Endergebnis.

Eine definierte Menge von 5 $\beta$-Pregnantriol- $(3 \alpha, 17 \alpha, 20 \alpha)$, die einem Harn zugesetzt wurde, wurde annähernd quantitativ oxydiert und beeinflußte die gefundene Pregnandiolausscheidung

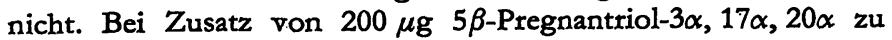
$90 \mathrm{ml}$ Harn betrug der ursprüngliche Pregnandiol-Gehalt des Harns $662 \mu \mathrm{g}$, der Mittelwert nach Zusatz des Pregnantriols wurde zu $668 \mu \mathrm{g}$ gefunden.

I. Chromatograpbie: Abweichend von der Originalvorschrift bringen wir den Toluolextrakt vollständig zur Trockne (Wasserbad, Stickstoffstrom) und nehmen den Trockenrückstand mit $5 \mathrm{ml}$. Toluol wieder auf. Um sicherzustellen, daß Lösungsmittelreste oder Wasserspuren, die die nachfolgende Chromatographie außerordentlich stören würden, entfernt werden, wird die getrocknete Probe über Nacht im Exsikkator $\left(\mathrm{CaCl}_{2}\right)$ aufbewahrt. Der Toluolextrakt kann nach unseren Erfahrungen in verschlossenen Kolben wochenlang aufbewahrt werden, ohne daß Verluste auftreten. Die Einstellung des Aluminiumoxyds auf die geeignete Aktivitätsstufe beobachtet man leicht daran, daß bei Elution mit 0,8\%
Athanol in Benzol alle eluierbaren Chromogene durchlaufen und daß der Durchlauf der Elution mit 3\% Athanol in Benzol farblos ist. Ist die Tropfgeschwindigkeit zu gering, so darf man sie durch Anwendung eines schwachen Druckes (Stickstoff) erhöhen.

Acetyliertung: Nach beendeter Umsetzung kann das überschüssige Acetylchlorid durch einfache Destillation aus dem Reaktionsgemisch entfernt werden. Das Ausschütteln mit Wasser und Neutralisieren mit Natriumbikarbonatlösung ist dann entbehrlich und damit eine erhebliche Arbeitsersparnis erreicht. Die Prüfung des $\mathrm{pH}-\mathrm{Wertes}$ der Extrakte zeigte stets eine neutrale Reaktion, so $\mathrm{da} \beta$ auch die laufende $\mathrm{pH}-$ Kontrolle entbehrlich ist.

II. Chromatograpbie: Es ist zweckmäßig, hier mit höhersiedendem Petroläther zu arbeiten, da dann die Gefahr der Bildung von Luftbläschen in der Säule und des Zerreißens der Säule wesentlich eingeschränkt ist. Nach Anfärbung mit Schwefelsäure sind die Eluate meist schon visull $z u$ beurteilen; eine spektrophotometrische Messung aller Eluate wird von uns nur dann durchgeführt, wenn eine neue Aluminiumoxydcharge zur Verwendung kommt. In der Routineanalyse ist sie entbehrlich.

Farbreaktion: Die vor der Anfärbung zuzusetzende Natriumsulfitmenge von $10 \mathrm{mg}$ wird von uns mit einem Kunststoffspatel, der eine kleine Vertiefung trägt, die etwa $10 \mathrm{mg}$ faßt, abgemessen; so spart man das Einwägen. Eine Versuchsreihe, bei der wir $10 \pm 4 \mathrm{mg}$ eingesetzt haben, zeigt, daß die Sulfitmenge keinen wesentlichen Einfluß auf die gemessene Extinktion hat. In der Meßreihe der Abbildung 1 wurden $200 \mu \mathrm{g}$ Pregnandiol ohne vorherige Acetylierung angefärbt. Es wurde einmal Meßprobe zind Bezugsprobe mit steigenden Mengen $\mathrm{Na}_{2} \mathrm{SO}_{3}$ angesetzt (.-_. $)$, das andere $\mathrm{Mal}$ nur in der $\mathrm{Me}$ probe steigende $\mathrm{Na}_{2} \mathrm{SO}_{3}$-Mengen gegen eine konstante Sulfitmenge in der Bezugsprobe angesetzt $(\cdot---\cdot)$.

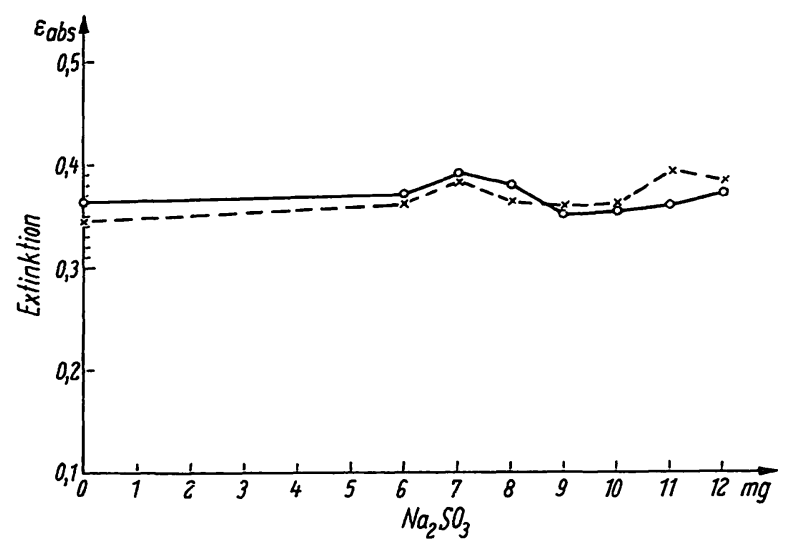

Abb. 1

Einfluß der $\mathrm{Na}_{2} \mathrm{SO}_{3}$-Menge auf die Extinktion des PregnandiolFarbkomplexes

Bezüglich der Entwicklung des Pregnandiol-Schwefelsäurefarbkomplexes versuchten wir, die in der Originalvorschrift geforderte 17-stdg. Entwicklungszeit bei $25^{\circ}$ abzukürzen. Wie die Abbildung 2 zeigt, läßt sich auch bei erhöhter Entwicklungstemperatur diese Zeitspanne nicht wesentlich verkürzen. Erst nach 17 Stunden ist die Zunahme der Extinktion mit der Zeit so gering, daß sie für die Dauer der Messung vernachlässigt werden kann (Abb. 2). Messung: Als Bezugslösung für die Messung lassen wir stets den Acetylchlorid/Benzol-Leeransatz mitlaufen. Nur so wird mit Sicherheit eine evtl. auf die Acetylierung zurückgehende Färbung eliminiert. (In den seltenen Fällen, in denen die Extinktionswerte zu hoch liegen, arbeiten wir mit Küvetten geringerer Schichtdicke. Sehr kleine Extinktionswerte, die die Verwendung von Küvetten größerer Schichtdicke erfordern würden, wurden von uns nie beobachtet.) Der Versuch einer Restfarbebestimmung brachte insofern kein Ergebnis, als nach Zerstörung der Pregnandiol-Gelbfärbung mittels $\mathrm{H}_{2} \mathrm{O}_{2}$ (3-proz.) keine meßbaren Färbungen auftreten. Es ist daher nicht möglich, eine unspezifische Färbung in Abzug zu bringen. 
ALLEN-Korrektur: Durch Aufnahme der Absorptionsspektren des authentischen $5 \beta$-Pregnandiols- $(3 \alpha, 20 \alpha)$ und des $5 \beta$-Pregnantriols- $(3 \alpha, 17 \alpha, 20 \alpha)$ bestimmten wir die Wellenlängen des Absorptionsmaximums (Abb. 3). Harnextrakte zeigen ein dem kristallinen Steroid ähnliches charakterisitisches Spektrum (Abb. 4).

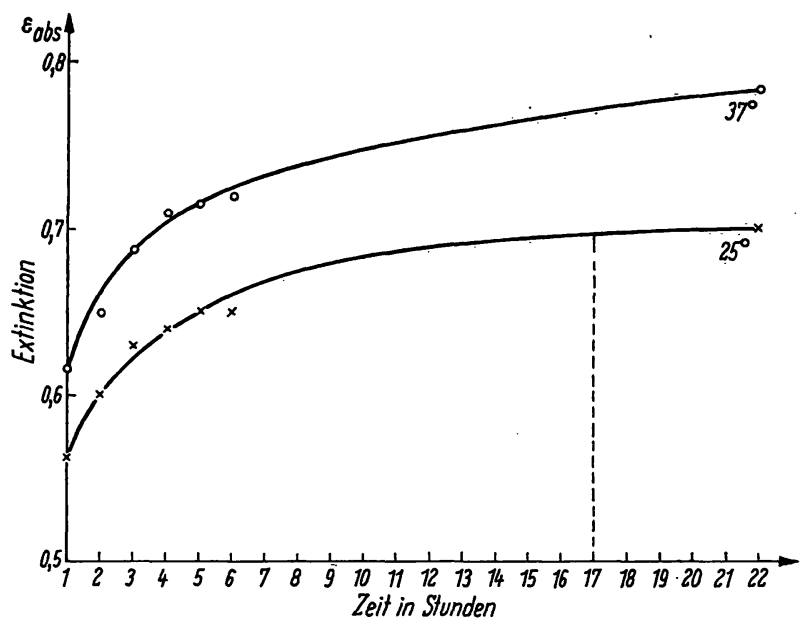

Abb. 2

Abhängigkeit der Extinktion des Pregnandiol-Farbkomplexes von Temperatur und Dauer der Farbentwicklung $(\lambda \max =430 \mathrm{~m} \mu)$

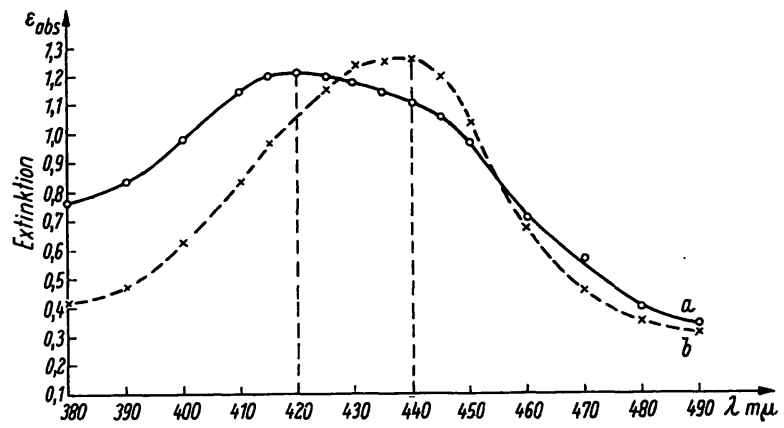

Abb. 3

Absorptionsspektren

a) des Pregnandiols- $3 \alpha, 20 \alpha ; \lambda \max .=420 \mathrm{~m} \mu(500 \mu \mathrm{g})$

b) des Pregnantriols-3 $\alpha, 17 \alpha, 20 \alpha ; \lambda \max .=440 \mathrm{~m} \mu(300 \mu \mathrm{g})$ nach Acetylierung

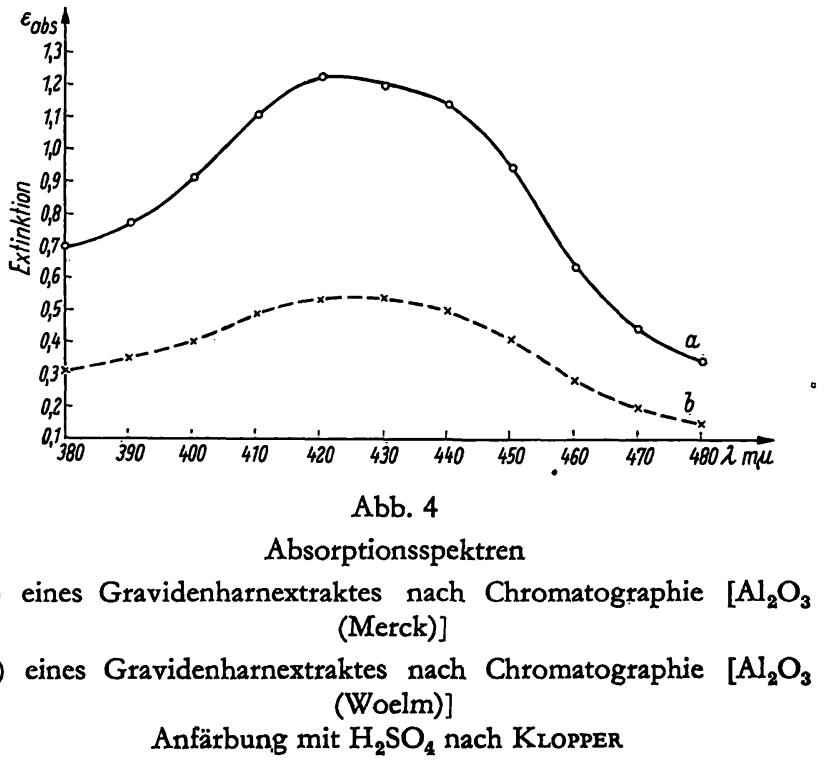

Tab. 1

Quantitativer Vergleich von 24-Stdn.-Pregnandiol-Ausscheidungswerten mit und ohne ALLEN-Korrektur

\begin{tabular}{|c|c|c|c|c|c|c|}
\hline Nr. & $\mathrm{E}_{430}$ & $\mathrm{E}_{\text {korr }}$ & $\begin{array}{l}\text { mg/24 Stdn. } \\
\text { ohne Korr. }\end{array}$ & $\begin{array}{c}\mathrm{mg} / 24 \mathrm{Stdn} . \\
\text { mit Korr. }\end{array}$ & $\begin{array}{c}\text { Differenz } \\
\Delta \mathrm{mg}\end{array}$ & $\begin{array}{c}\text { Differenz } \\
\Delta \%\end{array}$ \\
\hline 1. & 0,910 & 0,692 & 4,05 & 2,65 & $-1,40$ & $-34,6$ \\
\hline 2. & 0,499 & 0,238 & 2,25 & 1,75 & $-0,50$ & $-22,2$ \\
\hline 3. & 0,341 & 0,187 & 1,60 & 0,93 & $-0,67$ & $-41,9$ \\
\hline 4. & 0,430 & 0,271 & 2,20 & 1,65 & $-0,55$ & $-23,9$ \\
\hline 5. & 0,207 & 0,103 & 0,85 & 0,55 & $-0,30$ & $-35,3$ \\
\hline 6 & 0,601 & 0,378 & 2,35 & 1,88 & $-0,47$ & $-20,0$ \\
\hline 7. & 0,326 & 0,219 & 1,45 & 1,15 & $-0,30$ & $-20,7$ \\
\hline 8. & 0,690 & 0,505 & 2,93 & 2,58 & $-0,35$ & $-12,0$ \\
\hline 9. & 0,283 & 0,173 & 1,30 & 0,95 & $-0,35$ & $-26,9$ \\
\hline 10. & 0,174 & 0,078 & 0,70 & 0,35 & $-0,35$ & $-5 C, 0$ \\
\hline 11. & 0,875 & 0,655 & 3,95 & 3,55 & $-0,40$ & $-10,1$ \\
\hline 12. & 0,632 & 0,499 & 2,80 & 2,70 & $-0,10$ & $-3,8$ \\
\hline 13. & 0,242 & 0,142 & 1,10 & $\cdot 0,73$ & $-0,37$ & $-33,6$ \\
\hline 14. & 2,720 & 2 & 11,25 & 11 , & +0 & $+2,2$ \\
\hline 15. & 0,483 & 0 & 2,40 & 1,33 & $-1,07$ & $-44,6$ \\
\hline 16. & 0,341 & 0,1 & 1, & 3 & $-0,60$ & $-42,0$ \\
\hline 17. & 3, & & 12 & 11 & 0 & $-23,0$ \\
\hline 18. & 9 & & 23 & 5 & $-1,78$ & $-42,1$ \\
\hline 19. & 0,521 & 0, & 30 & 30 & $-1,00$ & $-43,5$ \\
\hline 20. & 1,000 & 0,627 & 4,50 & 3,30 & $-1,20$ & $-26,7$ \\
\hline 21. & 0,799 & 0,554 & 3,60 & 2,95 & $-0,65$ & $-18,1$ \\
\hline 22. & 0,590 & 0,254 & 2,65 & 1,35 & $-1,30$ & $-49,1$ \\
\hline 23. & 3,240 & 5,980 & 14,50 & 13,20 & $-1,30$ & $-9,0$ \\
\hline 24. & 3,960 & 3,480 & 17,80 & 18,40 & $+0,60$ & $+3,4$ \\
\hline 25. & 0,550 & 0,348 & 2,50 & 1,90 & $-0,60$ & $-24,0$ \\
\hline 26. & 0,472 & 0,278 & 2,10 & 1,50 & $-0,60$ & $-28,6$ \\
\hline 27. & 0,410 & 0,213 & 1,73 & 1,15 & $-0,58$ & $-33,5$ \\
\hline 28. & 0,276 & 0,154 & 1,32 & 0,95 & $-0,37$ & $-28,0$ \\
\hline 29. & 0,545 & 0,394 & 2,45 & 1,95 & $-0,50$ & $-20,4$ \\
\hline 30. & 1,035 & 0,692 & 4,75 & 3,30 & $-1,45$ & $-30,5$ \\
\hline 31. & 1,800 & 1,380 & $8 ; 0$ & 7,30 & $-0,70$ & $-8,8$ \\
\hline 32. & 0,850 & 0,658 & 3,50 & 3,17 & $-0,33$ & $-9,4$ \\
\hline 33. & 0,715 & 0,455 & 3,0 & 2,35 & $-0,68$ & $-22,4$ \\
\hline 34. & 0,685 & 0,2 & 2,80 & 2,25 & - & $-19,7$ \\
\hline 35. & 0 , & 0,128 & 20 & 5 & -0 & $-45,9$ \\
\hline 36. & 0,297 & 31 & & 0 & -0 & $-41,7$ \\
\hline 37. & 0,2 & 4 & 0,95 & 0 & - & $-47,4$ \\
\hline 38. & 0 , & 0 & 1,70 & 1,08 & - & $-36,5$ \\
\hline 39. & 0,605 & 0, & 2,75 & 2,10 & $-0,65$ & $-23,7$ \\
\hline 40. & 0,302 & 0,108 & 1,33 & 0,68 & $-0,65$ & $-48,9$ \\
\hline 41. & 0,688 & 0,459 & 3,10 & 2,45 & $-0,65$ & $-21,0$ \\
\hline 42. & 0,384 & 0,198 & 1,65 & 1,03 & $-0,62$ & $-37,6$ \\
\hline 43. & 1,010 & 0,724 & 4,80 & 4,10 & $-0,70$ & $-14,6$ \\
\hline 44. & 0,545 & 0,313 & 2,45 & 1,70 & $-0,75$ & $-30,6$ \\
\hline 45. & 0,476 & 0,231 & 2,15 & 1,25 & $-0,90$ & $-41,9$ \\
\hline 46. & 0,480 & 0,207 & 1,8 & 1,00 & $-0,85$ & $-43,6$ \\
\hline 47. & 0,415 & 0,2 & $1,9 ?$ & 1,23 & $-0,70$ & $-36,3$ \\
\hline 48. & 0,692 & 0,3 & 2,8 & 1,70 & $-1,13$ & $-36,4$ \\
\hline 49. & 0,399 & 0,214 & 1,73 & 1,03 & $-0,70$ & $-40,5$ \\
\hline 50. & 0,335 & 0,159 & 1,6 & 0,98 & $-0,67$ & $-40,6$ \\
\hline 51. & 0,690 & 0,379 & 3,0 & 2,13 & $-0,90$ & $-29,7$ \\
\hline 52. & 0,406 & 0,223 & 18 & 120 & $-0,65$ & $-35,1$ \\
\hline 53. & 0,620 & & & & $-0,70$ & $-25,5$ \\
\hline 54. & 0,7 & & & 2 & $-0,58$ & $-16,5$ \\
\hline 55. & 0,678 & 0,461 & 05 & 2,45 & $-0,60$ & $-19,7$ \\
\hline 56. & 1,3 & & & 5 & $-0,45$ & $-7,8$ \\
\hline 57. & 0,215 & 0 & 1,2 & 0,95 & $-0,30$ & $-24,0$ \\
\hline 58. & 0,565 & 0,237 & 2,55 & 1,25 & $-1,30$ & $-51,0$ \\
\hline 59. & 0,250 & 0,158 & 1,28 & 0,98 & $-0,30$ & $-23,5$ \\
\hline 60. & 0,497 & 0,289 & 2,10 & 1,63 & $-0,47$ & $-22,4$ \\
\hline 61. & 0,340 & 0,223 & 1,65 & 1,33 & $-0,32$ & $-19,4$ \\
\hline 62. & 0,424 & 0,287 & 1,95 & 1,50 & $-0,45$ & $-23,0$ \\
\hline 63. & 0,302 & 0,181 & 1,55 & 1,05 & $-0,50$ & 32,3 \\
\hline 64. & 0,618 & 0,389 & 2,75 & 2,10 & $-0,65$ & $-23,7$ \\
\hline 65. & 0,663 & 0,375 & 2,65 & 1,85 & $-0,80$ & $-30 ; 2$ \\
\hline 66. & 0,942 & 0,640 & 4,05 & 3,40 & $-0,65$ & $-16,1$ \\
\hline
\end{tabular}


Dies ermöglicht in Anlehnung an eine Arbeit von Lipp (11) die Anwendung der Allenschen Korrekturformel auf den Pregnandiol-Farbkomplex. Die von Lrpp angewandte papierchromatographische Auftrennung der Pregnandiol-Eluate, die nach Klopper erhalten werden, sowie die erneute Chromatographie der papierchromatographisch erhaltenen Fraktionen erscheinen uns entbehrlich, wenn es sich nicht um extrem niedrige Pregnandiolgehalte handelt. Im Routinebetrieb belastet sie das ohnehin langwierige Analysenverfahren erheblich. Wir glauben, daß die Anwendung der Allenschen Korrektur die an sich bereits hochgereinigten Pregnandiolextrakte spezifisch zu erfasssen gestattet. Gegenüber der von KLOPPER angegebenen einfachen Messung bei $430 \mathrm{~m} \mu$ werden dann allerdings Werte erhalten, die bis z" 50\% unter den nicht korrigierten Werten liegen.

Wir haben in einer Versuchsreihe 66 Pregnandiolextrakte aus Frauenharnen sowohl nach Klopper bei $430 \mathrm{~m} \mu$ als auch bei 380,420 und $460 \mathrm{~m} \mu$ spektrophotometrisch gemessen und die auftretenden Differenzen in $\mathrm{mg}$ sowie in Prozenten des „KLOPper-Wertes“ verfolgt. Der arithmetische Mittelwert der absoluten Differenz der Proben beträgt $0,71 \mathrm{mg}$, die mittlere prozentuale Differenz 28,8\% (Tab. 1). Grundlage für die Auswertung. war die.mit ALLEN-Korrektur am authentischen Pregnandiol aufgenommene, korrigierte Eichkurve (Abb. 5). Der Wert der Allen-Korrektur

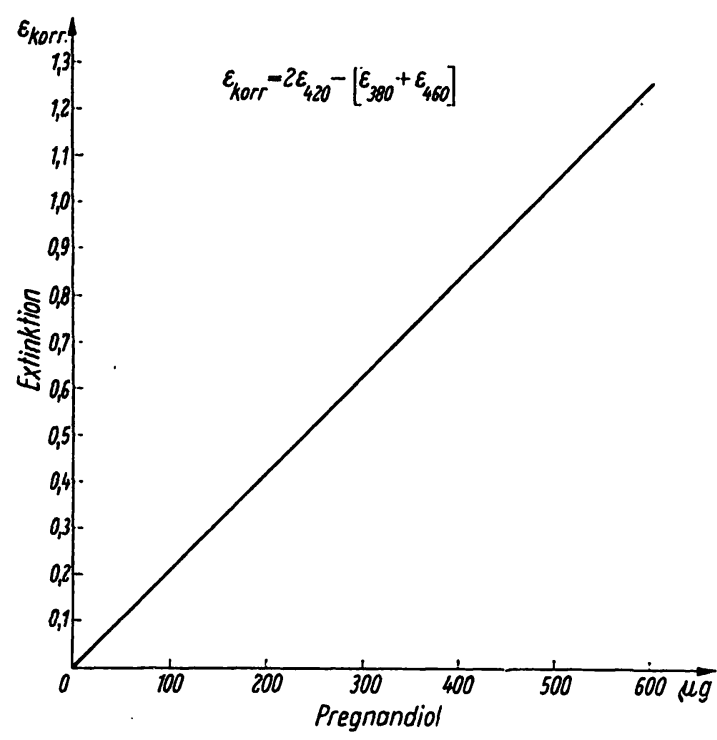

Abb. 5

Eichkurve des acetylierten Pregnandiols-3x,20x nach AllenKorrektur $\mathrm{E}_{\mathrm{korr}}=2 \mathrm{E}_{420}-\left(\mathrm{E}_{380}+\mathrm{E}_{460}\right)$

zeigt sich besonders deutlich an den hohen, „unwahrscheinlichen" Pregnandiol-Werten, die entweder bestätigt oder als Verunreinigung erkannt werden.

Meinen Mitarbeitern, insbesondere Fräulein MarianNe FahLKE, sage ich für ihre unermüdliche und gewissenhafte Mitarbeit meinen Dank.

\section{Literatur}

1. Klopper, A., E. A. Mrchie und J. B. Brown, J. Endocr. 12, 209 (1955). - 2. Venning, E. H., J. biol. Chemistry 119, 473 (1937). - 3. Venning, E. H., J. biol. Chemistry 126, 595 (1938). 4. Venning, E. H. und J. S. L. Browne, Endocrinology 27, 707 (1940). - 5. Shinowara, G. Y. und H. L. Reinhart, Amer. J. Clin. Path. 10, 77 (1940). - 6. Westrhal, U., Hoppe-Seyler's Z. physiol. Chem. 281, 14 (1944). - 7. Asrwoop, E. B. und G. E. S.
Jones, J. biol. Chemistry 137, 397 (1941). - 8. Talbot, N. B., R. A. Berman, E. A. Mclachlan und J. K. Wolfe, J. Clin. Endocr., Springfield 1, 668 (1941). - 9. Sommervirle, I. F., N. Govgr und G. F. Marrian, J. Endocr. 5, 247 (1948). 10. SMITH, O. W., J. Clin. Endocr., Springfield 10, 496 (1950). 11. Lipp, G., Acta endocr., K'hvn 33, 501 (1960). 